\title{
Suspended Mechanical Structures Based on Elastic Silicon Nanowire Arrays
}

\author{
Álvaro San Paulo, ,t,‡ Noel Arellano, ${ }^{\dagger}$ Jose A. Plaza, ${ }^{\S}$ Rongrui He," \\ Carlo Carraro, ${ }^{\perp}$ Roya Maboudian, ${ }^{\perp}$ Roger T. Howe, ${ }^{\dagger, \#}$ Jeff Bokor, ${ }^{\dagger}$ and \\ Peidong Yang"

\begin{abstract}
Department of Electrical Engineering and Computer Sciences, University of Campus UAB, Bellaterra 08193, Barcelona, Spain, Department of Chemistry, University of California, Berkeley, California 94720, and Department of Chemical Engineering, University of California, Berkeley, California 94720
\end{abstract} \\ California, Berkeley, California 94720, Centro Nacional de Microelectronica, CSIC,
}

Received December 8, 2006; Revised Manuscript Received February 15, 2007

\begin{abstract}
We demonstrate a bottom-up/top-down combined method for the fabrication of horizontally suspended, well-oriented and size-controlled Si nanowire arrays. Mechanical beamlike structures composed of multiple ordered arrays consecutively linked by transversal microspacers are obtained by this method. Such structures are used to investigate the mechanical elasticity of the nanowire arrays by atomic force microscopy. Our results point out important differences in the morphology and mechanical behavior of the fabricated nanowire-based structures with respect to equivalent bulk material structures.
\end{abstract}

The development of methods for the controlled assembly of semiconductor nanowires into well-ordered arrays is essential for the implementation of integrated electronic and electromechanical systems that exploit the unique properties of individual nanowires. ${ }^{1-7}$ On the other hand, nanowire arrays are also of fundamental interest as they exhibit novel properties different from that of bulk materials. ${ }^{8-10} \mathrm{Si}$ nanowire growth based on the vapor-liquid-solid (VLS) mechanism ${ }^{11-14}$ is one of the most efficient bottom-up fabrication alternatives explored so far for Si nanowire-based applications, ${ }^{15-20}$ as it provides a self-assembled growth of a large number of single-crystal nanowires with a high structural quality and a precise control over their properties. ${ }^{21-24}$

One of the most interesting properties of VLS-grown $\mathrm{Si}$ nanowires is revealed when they are horizontally synthesized between the opposing (111)-oriented sidewalls of microfabricated trenches on a Si substrate. ${ }^{24,25}$ Under these conditions,

\footnotetext{
* Corresponding author. E-mail: alvaro.sanpaulo@cnm.es. Phone: ++.34 .93 .594 .77 .00 ext.1213. Fax: ++.34 .93 .580 .14 .96 .

$\dagger$ Department of Electrical Engineering and Computer Sciences, University of California.

$¥$ Current address: Centro Nacional de Microelectronica, CSIC, Campus UAB, Bellaterra 08193, Barcelona, Spain.

$\S$ Centro Nacional de Microelectronica, CSIC, Campus UAB.

" Department of Chemistry, University of California.

${ }^{\perp}$ Department of Chemical Engineering, University of California.

\# Current address: Department of Electrical Engineering, Stanford University, Stanford, CA 94305.
}

the nanowires bridge the opposing sidewalls forming mechanically rigid and electrically continuous clamping points at both their base and impinging ends. ${ }^{3,24,25}$ So far, horizontal nanowire growth has been obtained for individual nanowires $^{3,6,24}$ and nanowire arrays ${ }^{25}$ without precise control of the location of the nanowires or the size of the arrays. Here we exploit the convenient features of horizontal growth for the fabrication of horizontal nanowire arrays with control of their size and location. We combine the VLS growth with conventional microfabrication techniques, which are used not only to fabricate the trenches but also to define specific nanowire growth areas at their sidewalls. As a result, we obtain mechanical structures which in the simplest case consist of an array clamped to the sidewall of a supporting structure at one end and to a freely suspended micropaddle at the other, resembling a single clamped microbeam with a body composed of a uniformly dense, well-oriented and sizecontrolled nanowire array instead of bulk $\mathrm{Si}$.

The fabrication process flow is shown in Figure 1. We start from silicon-on-insulator (SOI) wafers with a $2 \mu \mathrm{m}$ thick $\mathrm{Si}(110)$ device layer and a $0.5 \mu \mathrm{m}$ thick buried silicon oxide layer. ${ }^{24}$ First, the device layer is thermally oxidized to obtain a $160 \mathrm{~nm}$ thick oxide layer. Then, the trenches and the micropaddles are defined with (111)-oriented sidewalls by optical lithography and subsequent reactive ion etching of the top oxide and Si layers. A second thermal oxidation is 


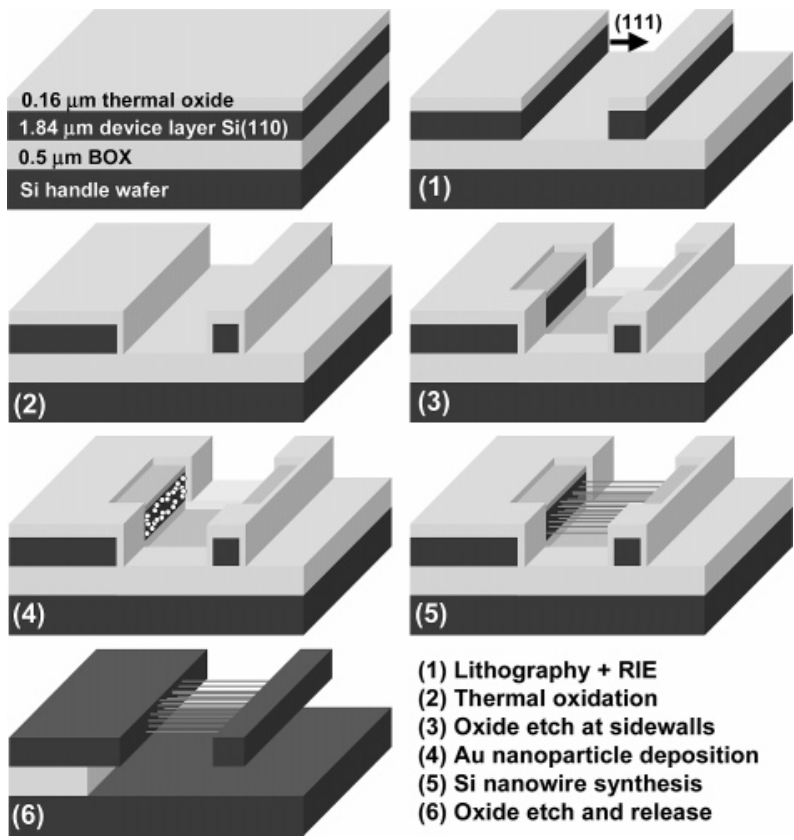

Figure 1. Fabrication process flow. Si nanowire growth locations at vertical sidewalls are lithographically defined in step 3. Diametercontrolled VLS growth only at these locations is achieved by using a galvanic displacement method for the Au catalyst nanoparticle deposition in step 4 .

performed next to obtain a $50 \mathrm{~nm}$ thick oxide layer on the sidewalls of the trenches. In the third step, a final lithography process delimits the areas at the sidewalls where the nanowires will grow. The oxide layer on these areas is removed by using 10:1 BHF and controlling the etching time so that the oxide is not removed from the top device layer. After that, all the substrate surface is covered by oxide except for the silicon exposed areas at the sidewalls where the nanowires are aimed to grow. The selective growth of nanowires only at these bare silicon areas is achieved by using a galvanic displacement method to deposit the $\mathrm{Au}$ catalyst nanoparticles needed for the VLS synthesis. The galvanic displacement method allows to control the $\mathrm{Au}$ nanoparticle diameter and it ensures nanoparticle deposition only at silicon exposed areas but not on silicon oxide covered surfaces. ${ }^{26,27}$ Undoped $\mathrm{Si}$ nanowires are then grown in a chemical vapor deposition tube furnace at $850{ }^{\circ} \mathrm{C}$ and atmospheric pressure. $\mathrm{SiCl}_{4}$ is used as precursor and $\mathrm{Ar} / \mathrm{H}_{2}(10 \%)$ is used as both the carrier and diluted gas (30$50 \mathrm{sccm}$ of $\mathrm{Ar} / \mathrm{H}_{2}(10 \%)$ was allowed to flow through a bubbler containing the liquid $\mathrm{SiCl}_{4}$ precursor and then to the tube, while $130-150 \mathrm{sccm}$ of $\mathrm{Ar} / \mathrm{H}_{2}(10 \%)$ was allowed to flow directly to the tube). ${ }^{24}$ As the Au catalyst nanoparticles determine the nanowire diameter and growth location, 1,2,21,27 nanowires grow only at the predetermined areas bridging the facing sidewalls of the supporting structure and micropaddle. Finally, the buried oxide layer is etched in 5:1 BHF and the structures are released in a high-pressure liquid carbon dioxide based supercritical point drying system.

A key step in the process is the galvanic displacement method used for the deposition of the Au catalyst nanoparticles. In this process, the Au nanoparticles are deposited by immersion of the substrates in a reversed micelle micro- emulsion that is prepared by mixing a water-based plating solution with $n$-heptane and a surfactant, sodium bis(2ethylhexyl) sulfosuccinate (AOT, $\left.\mathrm{C}_{20} \mathrm{H}_{37} \mathrm{O}_{7} \mathrm{SNa}\right)$. The waterbased solution consists of $0.2 \mathrm{M} \mathrm{HF}$ and $0.01 \mathrm{M} \mathrm{KAuCl}_{4}$. The AOT/heptane solution is made by dissolving $0.33 \mathrm{M}$ AOT in $n$-heptane. The two solutions are mixed to make microemulsions with different water-to-surfactant molar ratios $R=\left[\mathrm{H}_{2} \mathrm{O}\right] /[\mathrm{AOT}]$. The reversed micelles that are formed in the microemulsions contain the water-based solution and have a radius $R_{\mathrm{m}}$ that has been found to depend on $R$ according to the empiric $\operatorname{law}^{26} R_{\mathrm{m}}=0.175 R+1.5$. It has also been demonstrated that the diameter of the resulting $\mathrm{Au}$ nanoparticles is directly proportional to the diameter of the micelles, providing a way of controlling the diameter of the $\mathrm{Au}$ nanoparticles and consequently the diameter of the nanowires. ${ }^{27}$ In our case, we use a molar ratio $R=20$ to obtain an average diameter of the particles of $30 \pm 5 \mathrm{~nm}$ and a density of approximately 50 nanoparticles $\mu \mathrm{m}^{-2}$.

Figure 2 shows scanning electron microscopy (SEM) images of the obtained structures. Figure 2a displays the top view of a $5 \mu \mathrm{m}$ wide suspended array formed by $3 \mu \mathrm{m}$ long nanowires. The average diameter of the nanowires is $52 \pm$ $7 \mathrm{~nm}$ measured at their center. The nanowires bridge the sidewall of the supporting structure (left) with the sidewall of a $1 \mu \mathrm{m}$ wide micropaddle (right) that remains suspended after release (Figure 2b). The vast majority of nanowires grow horizontally along the $\langle 111\rangle$ direction perpendicular to the sidewalls and just in the region between the micropaddle and the supporting structure. Remarkably, the rigidity of the nanowires and the mechanical strength of their clamping points allow the array to hold the suspended micropaddle straight above the substrate, which indicates the absence of stress in the nanowires after growth. We have further pushed the ability of the nanowires to sustain suspended structures by using narrow microspacers to link up to ten consecutive nanowire arrays. Figure $2 \mathrm{c}$ shows four structures composed of ten linked arrays that also remain suspended straight above the substrate after release. In Figure $2 \mathrm{~d}$, the last three arrays of a structure are viewed from the bottom side. This image further demonstrates the highly selective control over the location of the arrays, grown exclusively between the sidewalls of consecutive spacers. It can be noted that no nanowires are grown at the front sidewall of the last spacer. This was intentionally done by not removing the oxide layer previously grown at this area, hence preventing the deposition of Au catalyst nanoparticles.

The characterization of the mechanical behavior of nanowire arrays is of great interest and importance for the development of nanowire-based electromechanical applications. ${ }^{28}$ We have fabricated a series of structures with a larger last micropaddle in order to test the mechanical elasticity of the horizontally suspended Si nanowire arrays with an atomic force microscope (AFM). These structures, shown in panels $\mathrm{a}-\mathrm{c}$ of Figure 3, are composed of one to five linked arrays of $5 \mu \mathrm{m}$ long nanowires with widths of 5,10, and $20 \mu \mathrm{m}$. Panels d and e of Figure 3 show closer views of the $20 \mu \mathrm{m}$ wide structure composed of five arrays. We obtain a uniform density of 55 nanowires per square micrometer with an 

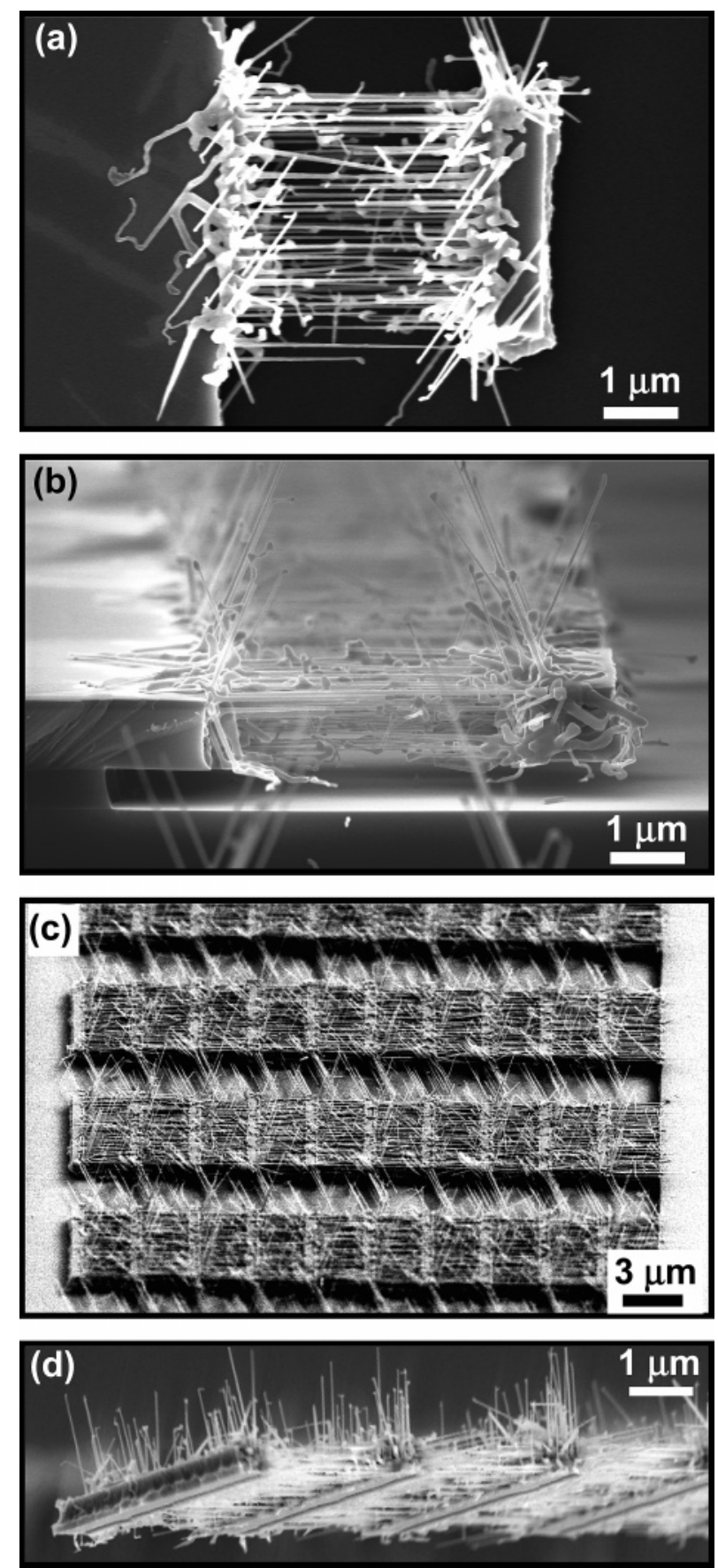

Figure 2. SEM Images of fabricated nanowire array structures. (a) Top view of a $3 \mu \mathrm{m}$ wide single suspended array structure composed of $3 \mu \mathrm{m}$ long nanowires with $50 \mathrm{~nm}$ diameter. (b) Cross section of a similar structure, showing the nanowires holding a suspended micropaddle. (c) Tilted view of a set of $10 \mu \mathrm{m}$ wide suspended structures composed of ten consecutive arrays linked by transversal spacers. (d) Bottom view of the three final arrays of a ten array structure.

average diameter of $50 \pm 6 \mathrm{~nm}$. Most of the nanowires bridge the opposing sidewalls, but we observe a small number of defects that can be classified in three types: horizontal nanowires that do not reach the opposing sidewall; nanowires oriented along out-of-plane $\langle 111\rangle$ directions; and nanowires that collide during their growth with nanowires growing in the opposite direction. We estimate the total number of defects to be lower than $10 \%$. Although some of the wider
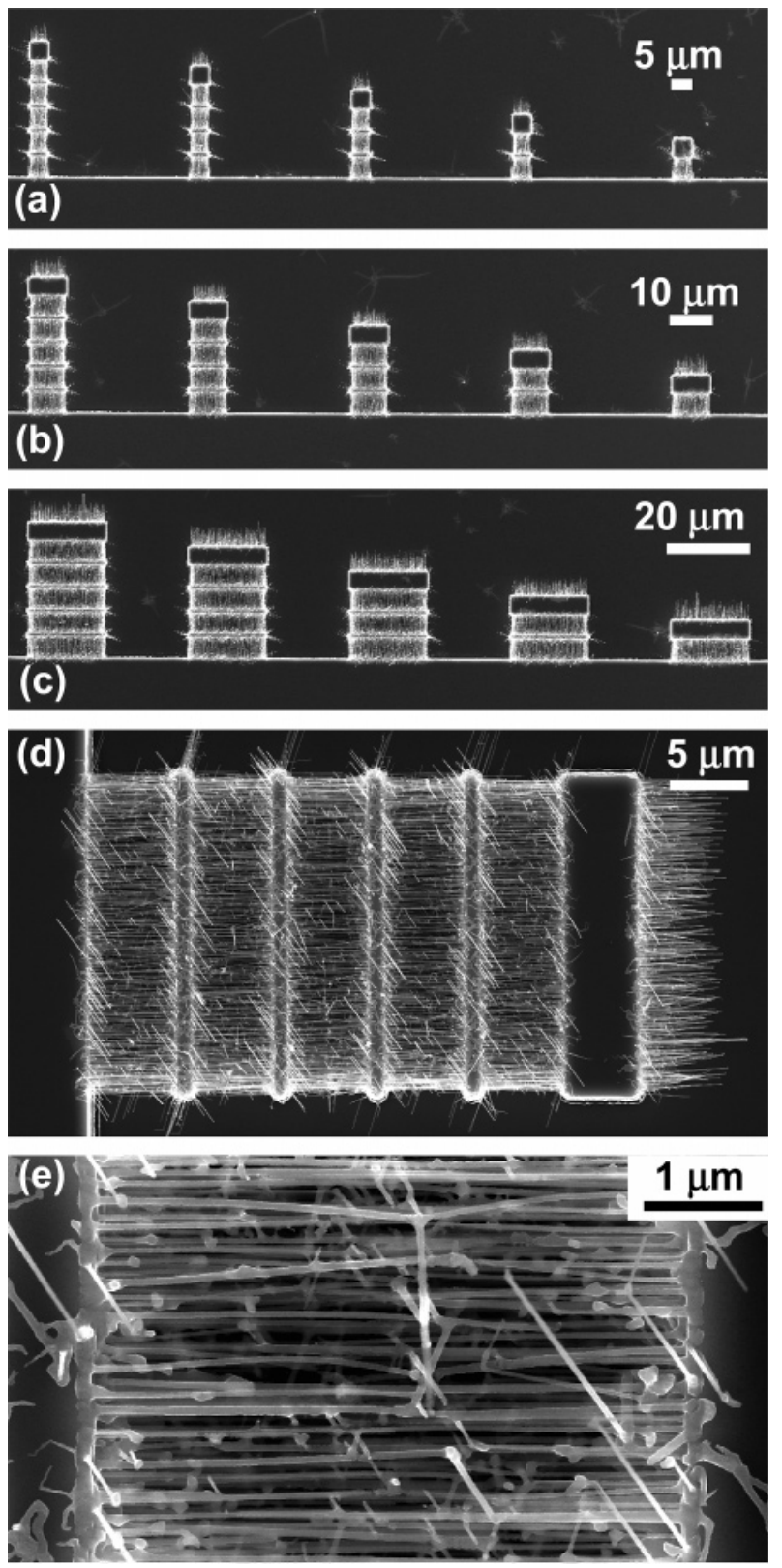

Figure 3. Structures fabricated for AFM mechanical characterization: (a) $5 \mu \mathrm{m}$ wide structures with one to five linked arrays of 5 $\mu \mathrm{m}$ long nanowires; (b) $10 \mu \mathrm{m}$ wide structures with one to five linked arrays; (c) $20 \mu \mathrm{m}$ wide structures with one to five linked arrays; (d) $20 \mu \mathrm{m}$ wide structure composed of 5 arrays of $5 \mu \mathrm{m}$ long nanowires; (e) detail of the center of a particular array, showing uniform density and distribution of diameters of the nanowires.

nanowires are slightly tapered, this effect seems significant only in secondary nanowires grown along out-of-plane $\langle 111\rangle$ directions. Such nanowires do not participate in linking consecutive arrays and hence do not contribute to the mechanical properties of the structures considered here.

In the experiments, the AFM tip (spring constant $2 \mathrm{~N} / \mathrm{m}$ ) was used to apply a controlled normal force on top of the center of the last suspended micropaddle in order to induce and measure the deflection of the structures. First, the AFM cantilever deflection was obtained as a function of the vertical AFM piezo displacement. Figure 4a shows the data obtained on a $5 \mu \mathrm{m}$ wide structure composed by five arrays. A slope 
(a)

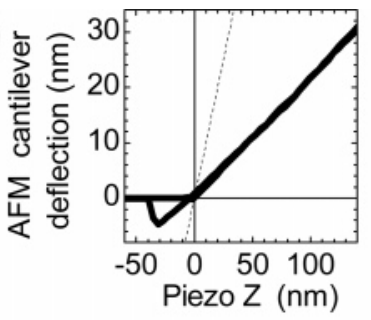

(b)

(c)

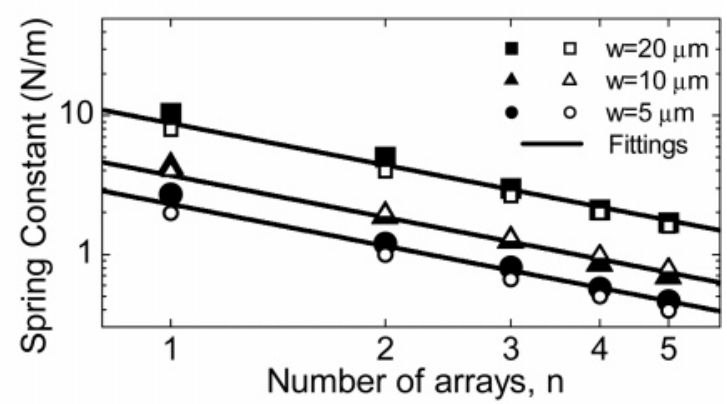

(d) Fixed

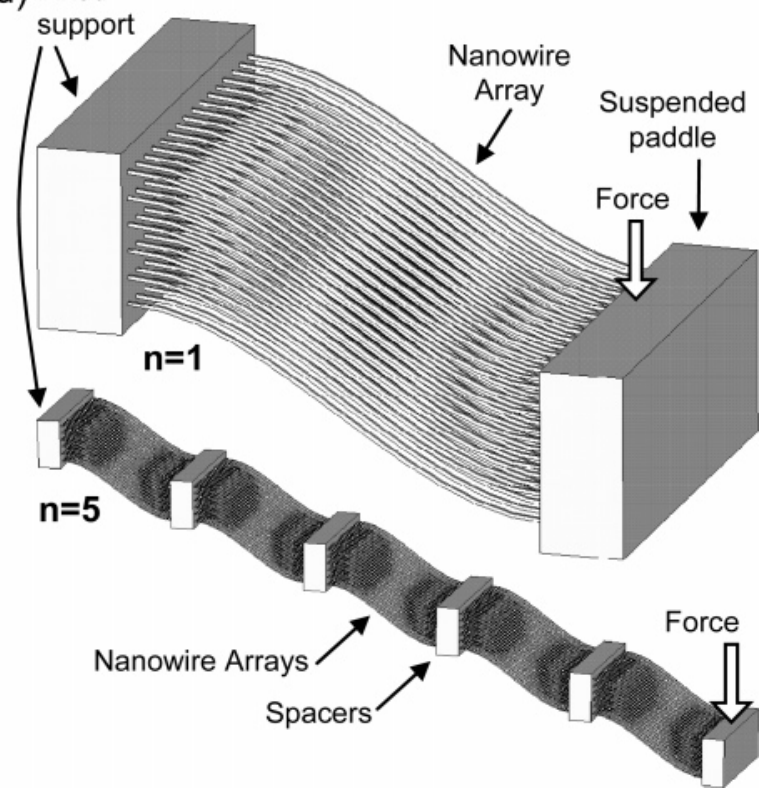

Figure 4. Mechanical testing and simulations of Si nanowire arrays. (a) AFM cantilever deflection vs piezo displacement curve obtained at the center of the large final micropaddle of a $5 \mu \mathrm{m}$ wide structure composed of five arrays of $5 \mu \mathrm{m}$ long nanowires. (b) Plot of the force applied by the AFM tip vs the deflection of the suspended microstructures obtained from the previous plot by subtracting the AFM cantilever deflection from the slope $=1$ line. (c) Spring constant vs number of arrays obtained by linear fittings of force vs deflection data. Solid symbols correspond to experimental results while hollow symbols represent data obtained from finite element simulations. (d) Finite element simulations of the deflection profile of the structures under the application of a normal force of $100 \mathrm{nN}$ on the last suspended paddle (the vertical displacement is $40 \times$ magnified in the graphs). The simulated arrays are composed of squared cross-section nanowires with the same density as the fabricated arrays and a width that matches the moment of inertia of cylindrical beams with a diameter equal to the experimental value.

lower than 1 in the region of positive cantilever deflection indicates the deflection of the structure, while the overlapping of the approaching and retracting curves shows the absence of detectable plastic deformation processes such as nanowires being broken or detached. ${ }^{3}$ By subtracting this portion of the plot from the line with slope $=1$, we obtain the deflection of the structure, which can be further plotted as a function of the force in order to calculate the corresponding spring constant $k$. In the previous case, a linear deflection of several tens of nanometers is observed with an applied force of several tens of nanonewtons (Figure $4 b$ ), resulting in a value of $k=0.45 \mathrm{~N} / \mathrm{m}$.

Similar results were obtained for all structures, which we summarize in Figure $4 \mathrm{c}$ by plotting the obtained spring constant values vs the number of consecutive arrays, $n$. In all cases, the best data fit corresponds to a $\sim 1 / n$ behavior. This is a rather different result from what would be expected by analogy with a bulk material microbeam, where the spring constant $k$ depends on its length $L$ as $k \sim 1 / L^{3}$. In our case, the proportionality between $n$ and $L$ should lead to a $\sim 1 / n^{3}$ dependence. The observed $\sim 1 / n$ behavior indicates that the spring constant of the structures is given by the contribution in series of each array, which contributes equally to the total deflection of the structure so that $k_{\text {total }}=k_{\text {array }} / n$, where $k_{\text {array }}$ is the effective spring constant of each array. We have investigated this behavior by performing finite element simulations of the deflection of similar array-based structures under the application of a normal force on the last paddle (Figure 4d). The simulations show a very interesting behavior of the nanowires, which bend parallel to each other keeping both ends flat and the sidewalls of each spacer parallel to each other. As a consequence, each array deflects equally as in the case of several identical linear springs linked in series and produces the observed $\sim 1 / n$ dependence of the total spring constant. The simulated values of $k$ are plotted by hollow symbols in Figure 4c. Besides reproducing the $\sim 1 / n$ behavior observed experimentally, the simulated data are in very good quantitative agreement with the experimental values.

To a first-order approximation, the elastic behavior of the arrays can be analytically described by considering that each of the nanowires contributes in parallel to $k_{\text {array }}$ so that $k_{\text {array }}$ $=N k_{\mathrm{nw}}$, where $N$ is the total number of nanowires. The spring constant of a nanowire (bending while keeping both ends flat) is written as $k_{\mathrm{nw}}=12 E I / /^{3}$, where $E$ is Young's modulus, $l$ is the length, and $I$ is the moment of inertia $I=\pi \mathrm{r}^{4} / 4(r=$ nanowire radius). With the measured density and dimensions of the nanowires and using $E=169 \mathrm{GPa}$ for $\mathrm{Si}(111),{ }^{3}$ we estimate spring constant values of $k_{\text {array }}=8.3,4.2$, and 2.1 $\mathrm{N} / \mathrm{m}$ for the 20,10 , and $5 \mu \mathrm{m}$ wide arrays, respectively. By fitting the experimental data to the expression $k_{\text {total }}=$ $k_{\text {array }} / n$, we obtain experimental values for $k_{\text {array }}$ of 8.8, 3.6, and $2.3 \mathrm{~N} / \mathrm{m}$ for the 20,10 , and $5 \mu \mathrm{m}$ wide arrays, respectively, again in very good agreement with the previous estimation.

The morphological and mechanical properties of the fabricated array-based structures present additional significant differences with respect to conventional bulk material beam structures. For instance, consider the $20 \mu \mathrm{m}$ wide structure with $n=5$ imaged in Figure 3d. The spring constant of a single-clamped Si beam with the same overall dimensions would be as large as $183 \mathrm{~N} / \mathrm{m}$. The measured spring constant is $1.7 \mathrm{~N} / \mathrm{m}, 2$ orders of magnitude smaller. In addition, the 
arrays also offer an important surface-to-volume enhancement with respect to bulk material structures. With the obtained density and nanowire dimensions, we estimate that each of the arrays represents a mass reduction of a factor of 8 and a surface increase of a factor of 10 with respect to the equivalent volume of bulk material; this makes a surfaceto-volume enhancement of a factor of 80, almost 2 orders of magnitude.

The ability to control the unique morphological and mechanical properties of Si nanowire arrays demonstrated here opens new perspectives for the development of arraybased electrical and electromechanical systems. Doubleclamped nanowire arrays represent an ideal system for the development of surrounding gate horizontal nanowire array FETs, ${ }^{10}$ as the epitaxial nanowire clamps provide excellent electrical contacts to source and drain electrodes. Suspended arrays could also be components of ultrasensitive piezoresistive transducers ${ }^{29}$ that would benefit from the extraordinarily enhanced piezoresistivity recently observed in Si nanowires. ${ }^{6}$ Also, a microcantilever sensor platform ${ }^{30}$ based on arrays could offer a superior sensitivity for mass or force sensing given the reduced mass and spring constant of the arrays, which would also provide a much larger area for the adsorption of particles or molecular recognition events in chemical or biological sensors.

Our results can be summarized in three points. First, we demonstrate a novel nanowire array fabrication process that allows simultaneous control over the array size and location, as well as over the nanowire orientation, diameter, and length. Second, we obtain unique beamlike mechanical structures composed of multiple arrays that show the ability of the asgrown nanowires to support horizontally suspended microstructures. Finally, we investigate the elasticity of the arrays by AFM. Under the application of a normal force, a suspended structure composed of consecutively linked arrays behaves like a set of linear springs connected in series instead of like a solid beam with the same overall dimensions. The resulting spring constant can indeed be more than 2 orders of magnitude lower than that of a bulk Si beam with the same overall dimensions, whereas the surface-to-volume ratio is enhanced by almost 2 orders of magnitude.

Acknowledgment. We thank E. Quevy, E. Lora-Tamayo, and G. Villanueva for very helpful discussions. This work was supported by National Science Foundation (Grant No. EEC-0425914).

\section{References}

(1) Wu, Y. Y.; Yang, P. D. J. Am. Chem. Soc. 2001, 123, 3165.

(2) Wu, Y. Y.; Fan, R.; Yang, P. D. Nano Lett. 2002, 2, 83.

(3) San Paulo, A.; Bokor, J.; Howe, R. T.; He, R.; Yang, P.; Gao, D.; Carraro, C.; Maboudian, R. Appl. Phys. Lett. 2005, 87, 3.

(4) Hoffmann, S.; Utke, I.; Moser, B.; Michler, J.; Christiansen, S. H.; Schmidt, V.; Senz, S.; Werner, P.; Gosele, U.; Ballif, C. Nano Lett. 2006, 6, 622 .

(5) Cui, Y.; Zhong, Z. H.; Wang, D. L.; Wang, W. U.; Lieber, C. M. Nano Lett. 2003, 3, 149.

(6) He, R.; Yang, P. Nat. Nanotechnol. 2006, 1, 42.

(7) Li, D. Y.; Wu, Y. Y.; Kim, P.; Shi, L.; Yang, P. D.; Majumdar, A. Appl. Phys. Lett. 2003, 83, 2934.

(8) Law, M.; Greene, L. E.; Johnson, J. C.; Saykally, R.; Yang, P. D. Nat. Mater. 2005, 4, 455.

(9) Lexholm, M.; Hessman, D.; Samuelson, L. Nano Lett. 2006, 6, 862.

(10) Goldberger, J.; Hochbaum, A. I.; Fan, R.; Yang, P. D. Nano Lett. 2006, 6, 973.

(11) Wagner, R. S.; Ellis, W. C. Appl. Phys. Lett. 1964, 4, 89.

(12) Westwater, J.; Gosain, D. P.; Tomiya, S.; Usui, S.; Ruda, H. J. Vac. Sci. Technol. 1997, 15, 554.

(13) Schmidt, V.; Senz, S.; Gosele, U. Nano Lett. 2005, 5, 931.

(14) Hannon, J. B.; Kodambaka, S.; Ross, F. M.; Tromp, R. M. Nature 2006, 440,69

(15) Wang, W. U.; Chen, C.; Lin, K. H.; Fang, Y.; Lieber, C. M. Proc. Natl. Acad. Sci. U.S.A. 2005, 102, 3208.

(16) Karnik, R.; Fan, R.; Yue, M.; Li, D. Y.; Yang, P. D.; Majumdar, A. Nano Lett. 2005, 5, 943.

(17) Ahn, Y.; Dunning, J.; Park, J. Nano Lett. 2005, 5, 1367.

(18) Schmidt, V.; Riel, H.; Senz, S.; Karg, S.; Riess, W.; Gosele, U. Small 2006, $2,85$.

(19) Zheng, G. F.; Lu, W.; Jin, S.; Lieber, C. M. Adv. Mater. 2004, 16, 1890.

(20) Zhong, Z. H.; Fang, Y.; Lu, W.; Lieber, C. M. Nano Lett. 2005, 5, 1143 .

(21) Hochbaum, A. I.; Fan, R.; He, R. R.; Yang, P. D. Nano Lett. 2005, $5,457$.

(22) Fuhrmann, B.; Leipner, H. S.; Hoche, H. R.; Schubert, L.; Werner, P.; Gosele, U. Nano Lett. 2005, 5, 2524.

(23) Yang, C.; Zhong, Z. H.; Lieber, C. M. Science 2005, 310, 1304.

(24) He, R. R.; Gao, D.; Fan, R.; Hochbaum, A. I.; Carraro, C.; Maboudian, R.; Yang, P. D. Adv. Mater. 2005, 17, 2098.

(25) Islam, M. S.; Sharma, S.; Kamins, T. I.; Williams, R. S. Nanotechnology 2004, 15, L5.

(26) Magagnin, L.; Bertani, V.; Cavallotti, P. L.; Maboudian, R.; Carraro, C. Microelectron. Eng. 2002, 64, 479 .

(27) Gao, D.; He, R. R.; Carraro, C.; Howe, R. T.; Yang, P. D.; Maboudian, R. J. Am. Chem. Soc. 2005, 127, 4574.

(28) Ekinci, K. L.; Roukes, M. L. Rev. Sci. Instrum. 2005, 76.

(29) Arlett, J. L.; Maloney, J. R.; Gudlewski, B.; Muluneh, M.; Roukes, M. L. Nano Lett. 2006, 6, 1000.

(30) Lavrik, N. V.; Sepaniak, M. J.; Datskos, P. G. Rev. Sci. Instrum. 2004, 75, 2229.

NL062877N 